\title{
ON NEW INEQUALITIES OF SIMPSON'S TYPE FOR FUNCTIONS WHOSE SECOND DERIVATIVES ABSOLUTE VALUES ARE CONVEX
}

MEHMET ZEKI SARIKAYA, ERHAN. SET AND M. EMIN OZDEMIR

\begin{abstract}
In this note, we obtain new some inequalities of Simpson's type based on convexity. Some applications for special means of real numbers are also given.
\end{abstract}

Mathematics Subject Classification 2000: 26D15, 26D10

Additional Key Words and Phrases: Simpson's inequality, convex function

\section{INTRODUCTION}

The following Theorem describes the known [3] in the literature as Simpson's inequality.

THEOREM 1.1. Let $f:[a, b] \rightarrow \mathbb{R}$ be a four times continuously differentiable mapping on $(a, b)$ and $\left\|f^{(4)}\right\|_{\infty}=\sup _{x \in(a, b)}\left|f^{(4)}(x)\right|<\infty$. Then, the following inequality holds:

$$
\left|\frac{1}{3}\left[\frac{f(a)+f(b)}{2}+2 f\left(\frac{a+b}{2}\right)\right]-\frac{1}{b-a} \int_{a}^{b} f(x) d x\right| \leq \frac{1}{2880}\left\|f^{(4)}\right\|_{\infty}(b-a)^{4} .
$$

For recent refinements, counterparts, generalizations and new Simpson's type inequalities, see ([2],[3],[5]).

In [3], Dragomir et. al. proved the following some recent developments on Simpson's inequality for which the remainder is expressed in terms of lower derivatives than the fourth.

TheOREM 1.2. Suppose $f:[a, b] \rightarrow \mathbb{R}$ is a differentiable mapping whose derivative is continuous on $(a, b)$ and $f^{\prime} \in L[a, b]$. Then the following inequality

$$
\left|\frac{1}{3}\left[\frac{f(a)+f(b)}{2}+2 f\left(\frac{a+b}{2}\right)\right]-\frac{1}{b-a} \int_{a}^{b} f(x) d x\right| \leq \frac{b-a}{3}\left\|f^{\prime}\right\|_{1}
$$

holds, where $\left\|f^{\prime}\right\|_{1}=\int_{a}^{b}\left|f^{\prime}(x)\right| d x$.

The bound of (1) for L-Lipschitzian mapping was given in [3] by $\frac{5}{36} L(b-a)$.

Also, the following inequality was obtained in [3]. 
TheOREM 1.3. Suppose $f:[a, b] \rightarrow \mathbb{R}$ is an absolutely continuous mapping on $[a, b]$ whose derivative belongs to $L_{p}[a, b]$. Then the following inequality holds,

$$
\begin{gathered}
\left|\frac{1}{3}\left[\frac{f(a)+f(b)}{2}+2 f\left(\frac{a+b}{2}\right)\right]-\frac{1}{b-a} \int_{a}^{b} f(x) d x\right| \leq \\
\leq \frac{1}{6}\left[\frac{2^{q+1}+1}{3(q+1)}\right]^{\frac{1}{q}}(b-a)^{\frac{1}{q}}\left\|f^{\prime}\right\|_{p}
\end{gathered}
$$

where $\frac{1}{p}+\frac{1}{q}=1$.

In [1] Alomari et. al. obtained some inequalities for functions whose second derivatives absolute values are quasi-convex connecting with the Hermit-Hadamard inequality on the basis of the following Lemma.

Lemma 1.4. Let $f: I \subset \mathbb{R} \rightarrow \mathbb{R}$ be twice differentiable mapping on $I^{\circ}$ with $f^{\prime \prime} \in L_{1}[a, b]$, then

$$
\frac{f(a)+f(b)}{2}-\frac{1}{b-a} \int_{a}^{b} f(x) d x=\frac{(b-a)^{2}}{2} \int_{0}^{1} t(1-t) f^{\prime \prime}(t a+(1-t) b) d t .
$$

In [4], Hussain et. al. prove some inequalities related to Hermite-Hadamard's inequality for $s$-convex functions by used the above lemma.

TheOrem 1.5. Let $f: I \subset[0, \infty) \rightarrow \mathbb{R}$ be twice differentiable mapping on $I^{\circ}$ such that $f^{\prime \prime} \in L_{1}[a, b]$ where $a, b \in I$ with $a<b$. If $\left|f^{\prime \prime}\right|$ is $s$-convex on $[a, b]$ for some fixed $s \in[0,1]$ and $q \geq 1$, then the following inequality holds:

$$
\left|\frac{f(a)+f(b)}{2}-\frac{1}{b-a} \int_{a}^{b} f(x) d x\right| \leq \frac{(b-a)^{2}}{2 \times 6^{\frac{1}{p}}}\left[\frac{\left|f^{\prime \prime}(a)\right|^{q}+\left|f^{\prime \prime}(b)\right|^{q}}{(s+2)(s+3)}\right]^{\frac{1}{q}}
$$

where $\frac{1}{p}+\frac{1}{q}=1$

Remark 1.6. If we take $s=1$ in (4), then

$$
\left|\frac{f(a)+f(b)}{2}-\frac{1}{b-a} \int_{a}^{b} f(x) d x\right| \leq \frac{(b-a)^{2}}{12}\left[\frac{\left|f^{\prime \prime}(a)\right|^{q}+\left|f^{\prime \prime}(b)\right|^{q}}{2}\right]^{\frac{1}{q}} .
$$

The main aim of this paper is to establish new Simpson's type inequalities for the class of functions whose derivatives in absolute value at certain powers are convex functions.

\section{MAIN RESULTS}

In order to prove our main theorems, we need the following Lemma.

Lemma 2.1. Let $f: I \subset \mathbb{R} \rightarrow \mathbb{R}$ be twice differentiable mapping on $I^{\circ}$ such that $f^{\prime \prime} \in L_{1}[a, b]$, where $a, b \in I$ with $a<b$, then the following equality holds:

$$
\begin{aligned}
& \frac{1}{6}\left[f(a)+4 f\left(\frac{a+b}{2}\right)+f(b)\right]-\frac{1}{b-a} \int_{a}^{b} f(x) d x \\
& =(b-a)^{2} \int_{0}^{1} k(t) f^{\prime \prime}(t b+(1-t) a) d t
\end{aligned}
$$


where

$$
k(t)= \begin{cases}\frac{t}{2}\left(\frac{1}{3}-t\right), & t \in\left[0, \frac{1}{2}\right) \\ (1-t)\left(\frac{t}{2}-\frac{1}{3}\right), & t \in\left[\frac{1}{2}, 1\right] .\end{cases}
$$

Proof. By definition of $k(t)$, we have

$$
\begin{aligned}
& I=\int_{0}^{1} k(t) f^{\prime \prime}(t b+(1-t) a) d t \\
& =\int_{0}^{\frac{1}{2}} \frac{t}{2}\left(\frac{1}{3}-t\right) f^{\prime \prime}(t b+(1-t) a) d t+\int_{\frac{1}{2}}^{1}(1-t)\left(\frac{t}{2}-\frac{1}{3}\right) f^{\prime \prime}(t b+(1-t) a) d t \\
& =I_{1}+I_{2} .
\end{aligned}
$$

Integrating by parts twice, we can state:

$$
\begin{aligned}
& I_{1}=-\frac{1}{24(b-a)} f^{\prime}\left(\frac{a+b}{2}\right)+\frac{1}{b-a} \int_{0}^{\frac{1}{2}}\left(t-\frac{1}{6}\right) f^{\prime}(t b+(1-t) a) d t \\
& =-\frac{1}{24(b-a)} f^{\prime}\left(\frac{a+b}{2}\right)+\frac{1}{(b-a)^{2}}\left[\frac{1}{3} f\left(\frac{a+b}{2}\right)+\frac{1}{6} f(a)-\int_{0}^{\frac{1}{2}} f(t b+(1-t) a) d t\right]
\end{aligned}
$$

and similarly,

$$
\begin{aligned}
& I_{2}=\frac{1}{24(b-a)} f^{\prime}\left(\frac{a+b}{2}\right)+\frac{1}{b-a} \int_{\frac{1}{2}}^{1}\left(t-\frac{5}{6}\right) f^{\prime}(t b+(1-t) a) d t \\
& =\frac{1}{24(b-a)} f^{\prime}\left(\frac{a+b}{2}\right)+\frac{1}{(b-a)^{2}}\left[\frac{1}{3} f\left(\frac{a+b}{2}\right)+\frac{1}{6} f(b)-\int_{\frac{1}{2}}^{1} f(t b+(1-t) a) d t\right] .
\end{aligned}
$$

Adding (8) and (9),

$$
\begin{aligned}
& I=I_{1}+I_{2} \\
& =\frac{1}{(b-a)^{2}}\left[\frac{1}{6} f(a)+\frac{2}{3} f\left(\frac{a+b}{2}\right)+\frac{1}{6} f(b)-\int_{0}^{1} f(t b+(1-t) a) d t\right] .
\end{aligned}
$$

Using the change of the variable $x=t b+(1-t)$ a for $t \in[0,1]$ and multiplying the both sides by $(b-a)^{2}$, we obtain (6) which completes the proof.

The next theorems give a new refinement of Simpson's inequality for twice differentiable functions:

THEOREM 2.2. Let $f: I \subset \mathbb{R} \rightarrow \mathbb{R}$ be twice differentiable mapping on $I^{\circ}$ such that $f^{\prime \prime} \in L_{1}[a, b]$, where $a, b \in I$ with $a<b$. If $\left|f^{\prime \prime}\right|$ is convex on $[a, b]$, then the following inequality holds:

$$
\left|\frac{1}{6}\left[f(a)+4 f\left(\frac{a+b}{2}\right)+f(b)\right]-\frac{1}{b-a} \int_{a}^{b} f(x) d x\right| \leq \frac{(b-a)^{2}}{162}\left[\left|f^{\prime \prime}(a)\right|+\left|f^{\prime \prime}(b)\right|\right] .
$$


Proof. From Lemma 2.1 and by using the convexity of $\left|f^{\prime \prime}\right|$, we get

$$
\begin{aligned}
& \left|\frac{1}{6}\left[f(a)+4 f\left(\frac{a+b}{2}\right)+f(b)\right]-\frac{1}{b-a} \int_{a}^{b} f(x) d x\right| \\
& \leq(b-a)^{2} \int_{0}^{1}|k(t)|\left|f^{\prime \prime}(t b+(1-t) a)\right| d t \\
& \leq(b-a)^{2}\left\{\int_{0}^{\frac{1}{2}}\left|\frac{t}{2}\left(\frac{1}{3}-t\right)\right|\left[t\left|f^{\prime \prime}(b)\right|+(1-t)\left|f^{\prime \prime}(a)\right|\right] d t\right. \\
& \left.+\int_{\frac{1}{2}}^{1}\left|(1-t)\left(\frac{t}{2}-\frac{1}{3}\right)\right|\left[t\left|f^{\prime \prime}(b)\right|+(1-t)\left|f^{\prime \prime}(a)\right|\right] d t\right\} \\
& =(b-a)^{2}\left(J_{1}+J_{2}\right)
\end{aligned}
$$

where

$$
J_{1}=\int_{0}^{\frac{1}{2}}\left|\frac{t}{2}\left(\frac{1}{3}-t\right)\right|\left[t\left|f^{\prime \prime}(b)\right|+(1-t)\left|f^{\prime \prime}(a)\right|\right] d t
$$

and

$$
J_{2}=\int_{\frac{1}{2}}^{1}\left|(1-t)\left(\frac{t}{2}-\frac{1}{3}\right)\right|\left[t\left|f^{\prime \prime}(b)\right|+(1-t)\left|f^{\prime \prime}(a)\right|\right] d t .
$$

By simple computation,

$$
\begin{aligned}
& J_{1}=\int_{0}^{\frac{1}{3}} \frac{t}{2}\left(\frac{1}{3}-t\right)\left[t\left|f^{\prime \prime}(b)\right|+(1-t)\left|f^{\prime \prime}(a)\right|\right] d t \\
& +\int_{\frac{1}{3}}^{\frac{1}{2}} \frac{t}{2}\left(t-\frac{1}{3}\right)\left[t\left|f^{\prime \prime}(b)\right|+(1-t)\left|f^{\prime \prime}(a)\right|\right] d t \\
& =\frac{59}{3^{5} 2^{7}}\left|f^{\prime \prime}(b)\right|+\frac{133}{3^{5} 2^{7}}\left|f^{\prime \prime}(a)\right|
\end{aligned}
$$

and

$$
\begin{aligned}
& J_{2}=\int_{\frac{1}{2}}^{\frac{2}{3}}(1-t)\left(\frac{1}{3}-\frac{t}{2}\right)\left[t\left|f^{\prime \prime}(b)\right|+(1-t)\left|f^{\prime \prime}(a)\right|\right] d t \\
& +\int_{\frac{2}{3}}^{1}(1-t)\left(\frac{t}{2}-\frac{1}{3}\right)\left[t\left|f^{\prime \prime}(b)\right|+(1-t)\left|f^{\prime \prime}(a)\right|\right] d t \\
& =\frac{133}{3^{5} 2^{7}}\left|f^{\prime \prime}(b)\right|+\frac{59}{3^{5} 2^{7}}\left|f^{\prime \prime}(a)\right|
\end{aligned}
$$

which completes the proof.

An immediate consequence of Theorem 2.2 is the following Corollary:

Corollary 2.3. Let $f: I \subset \mathbb{R} \rightarrow \mathbb{R}$ be twice differentiable mapping on $I^{\circ}$ such that $f^{\prime \prime} \in L_{1}[a, b]$, where $a, b \in I$ with $a<b$. If $f(a)=f\left(\frac{a+b}{2}\right)=f(b)$ and $\left|f^{\prime \prime}\right|$ is convex on $[a, b]$, then the following inequality holds:

$$
\left|\frac{1}{b-a} \int_{a}^{b} f(x) d x-f\left(\frac{a+b}{2}\right)\right| \leq \frac{(b-a)^{2}}{162}\left[\left|f^{\prime \prime}(a)\right|+\left|f^{\prime \prime}(b)\right|\right] .
$$

Remark 2.4. We note that the obtained midpoint inequality (10) is better than the inequality (1). 
A similar result is embodied in the following theorem.

TheOREM 2.5. Let $f: I \subset \mathbb{R} \rightarrow \mathbb{R}$ be twice differentiable mapping on $I^{\circ}$ such that $f^{\prime \prime} \in L_{1}[a, b]$, where $a, b \in I$ with $a<b$. If $\left|f^{\prime \prime}\right|^{q}$ is convex on $[a, b]$ and $q \geq 1$, then the following inequality holds:

$$
\begin{aligned}
& \left|\frac{1}{6}\left[f(a)+4 f\left(\frac{a+b}{2}\right)+f(b)\right]-\frac{1}{b-a} \int_{a}^{b} f(x) d x\right| \\
& \leq(b-a)^{2}\left(\frac{1}{162}\right)^{1-\frac{1}{q}} \\
& \left\{\left(\frac{59}{3^{5} 2^{7}}\left|f^{\prime \prime}(b)\right|^{q}+\frac{133}{3^{5} 2^{7}}\left|f^{\prime \prime}(a)\right|^{q}\right)^{\frac{1}{q}}+\left(\frac{133}{3^{5} 2^{7}}\left|f^{\prime \prime}(b)\right|^{q}+\frac{59}{3^{5} 2^{7}}\left|f^{\prime \prime}(a)\right|^{q}\right)^{\frac{1}{q}}\right\}
\end{aligned}
$$

where $\frac{1}{p}+\frac{1}{q}=1$.

Proof. Suppose that $q \geq 1$. From Lemma 2.1, we have

$$
\begin{aligned}
& \left|\frac{1}{6}\left[f(a)+4 f\left(\frac{a+b}{2}\right)+f(b)\right]-\frac{1}{b-a} \int_{a}^{b} f(x) d x\right| \\
& \leq(b-a)^{2} \int_{0}^{1}|k(t)|\left|f^{\prime \prime}(t b+(1-t) a)\right| d t \\
& =(b-a)^{2}\left\{\int_{0}^{\frac{1}{2}}\left|\frac{t}{2}\left(\frac{1}{3}-t\right)\right|\left|f^{\prime \prime}(t b+(1-t) a)\right| d t\right. \\
& \left.+\int_{\frac{1}{2}}^{1}\left|(1-t)\left(\frac{t}{2}-\frac{1}{3}\right)\right|\left|f^{\prime \prime}(t b+(1-t) a)\right| d t\right\} .
\end{aligned}
$$

Using the Hölder's inequality for functions

$$
\left|\frac{t}{2}\left(\frac{1}{3}-t\right)\right|^{1-\frac{1}{q}}
$$

and

$$
\left|\frac{t}{2}\left(\frac{1}{3}-t\right)\right|^{\frac{1}{q}}\left|f^{\prime \prime}(t b+(1-t) a)\right|
$$

for the first integral and the functions

$$
\left|(1-t)\left(\frac{t}{2}-\frac{1}{3}\right)\right|^{1-\frac{1}{q}}
$$

and

$$
\left|(1-t)\left(\frac{t}{2}-\frac{1}{3}\right)\right|^{\frac{1}{q}}\left|f^{\prime \prime}(t b+(1-t) a)\right|
$$


for the second integral, from the above relation we get the inequalities:

$$
\begin{aligned}
& \left|\frac{1}{6}\left[f(a)+4 f\left(\frac{a+b}{2}\right)+f(b)\right]-\frac{1}{b-a} \int_{a}^{b} f(x) d x\right| \\
& \leq(b-a)^{2}\left\{\left(\int_{0}^{\frac{1}{2}}\left|\frac{t}{2}\left(\frac{1}{3}-t\right)\right| d t\right)^{1-\frac{1}{q}}\left(\int_{0}^{\frac{1}{2}}\left|\frac{t}{2}\left(\frac{1}{3}-t\right)\right|\left|f^{\prime \prime}(t b+(1-t) a)\right|^{q} d t\right)^{\frac{1}{q}}\right. \\
& \left.+\left(\int_{\frac{1}{2}}^{1}\left|(1-t)\left(\frac{t}{2}-\frac{1}{3}\right)\right| d t\right)^{1-\frac{1}{q}}\left(\int_{\frac{1}{2}}^{1}\left|(1-t)\left(\frac{t}{2}-\frac{1}{3}\right)\right|\left|f^{\prime \prime}(t b+(1-t) a)\right|^{q} d t\right)^{\frac{1}{q}}\right\} .
\end{aligned}
$$

Since $\left|f^{\prime \prime}\right|^{q}$ is convex, therefore we have

$$
\begin{aligned}
& \int_{0}^{\frac{1}{2}}\left|\frac{t}{2}\left(\frac{1}{3}-t\right)\right|\left|f^{\prime \prime}(t b+(1-t) a)\right|^{q} d t \\
& \leq \int_{0}^{\frac{1}{2}}\left|\frac{t}{2}\left(\frac{1}{3}-t\right)\right|\left[t\left|f^{\prime \prime}(b)\right|^{q}+(1-t)\left|f^{\prime \prime}(a)\right|^{q}\right] d t \\
& =\int_{0}^{\frac{1}{3}}\left[\frac{t}{2}\left(\frac{1}{3}-t\right)\right]\left[t\left|f^{\prime \prime}(b)\right|^{q}+(1-t)\left|f^{\prime \prime}(a)\right|^{q}\right] d t \\
& +\int_{\frac{1}{3}}^{\frac{1}{2}}\left[\frac{t}{2}\left(t-\frac{1}{3}\right)\right]\left[t\left|f^{\prime \prime}(b)\right|^{q}+(1-t)\left|f^{\prime \prime}(a)\right|^{q}\right] d t \\
& =\frac{59}{3^{5} 2^{7}}\left|f^{\prime \prime}(b)\right|^{q}+\frac{133}{3^{5} 2^{7}}\left|f^{\prime \prime}(a)\right|^{q}
\end{aligned}
$$

and

$$
\begin{aligned}
& \int_{\frac{1}{2}}^{1}\left|(1-t)\left(\frac{t}{2}-\frac{1}{3}\right)\right|\left|f^{\prime \prime}(t b+(1-t) a)\right|^{q} d t \\
& \leq \int_{\frac{1}{2}}^{1}\left|(1-t)\left(\frac{t}{2}-\frac{1}{3}\right)\right|\left(t\left|f^{\prime \prime}(b)\right|^{q}+(1-t)\left|f^{\prime \prime}(a)\right|^{q}\right) d t \\
& =\int_{\frac{1}{2}}^{\frac{2}{3}}(1-t)\left(\frac{1}{3}-\frac{t}{2}\right)\left(t\left|f^{\prime \prime}(b)\right|^{q}+(1-t)\left|f^{\prime \prime}(a)\right|^{q}\right) d t \\
& +\int_{\frac{2}{3}}^{1}(1-t)\left(\frac{t}{2}-\frac{1}{3}\right)\left(t\left|f^{\prime \prime}(b)\right|^{q}+(1-t)\left|f^{\prime \prime}(a)\right|^{q}\right) d t \\
& =\frac{133}{3^{5} 2^{7}}\left|f^{\prime \prime}(b)\right|^{q}+\frac{59}{3^{5} 2^{7}}\left|f^{\prime \prime}(a)\right|^{q}
\end{aligned}
$$


From (11) and (12), we have

$$
\begin{aligned}
& \left|\frac{1}{6}\left[f(a)+4 f\left(\frac{a+b}{2}\right)+f(b)\right]-\frac{1}{b-a} \int_{a}^{b} f(x) d x\right| \\
& \leq(b-a)^{2}\left\{\left(\int_{0}^{\frac{1}{2}}\left|\frac{t}{2}\left(\frac{1}{3}-t\right)\right| d t\right)^{1-\frac{1}{q}}\left(\frac{59}{3^{5} 2^{7}}\left|f^{\prime \prime}(b)\right|^{q}+\frac{133}{3^{5} 2^{7}}\left|f^{\prime \prime}(a)\right|^{q}\right)^{\frac{1}{q}}\right. \\
& \left.+\left(\int_{\frac{1}{2}}^{1}\left|(1-t)\left(\frac{t}{2}-\frac{1}{3}\right)\right| d t\right)^{1-\frac{1}{q}}\left(\frac{133}{3^{5} 2^{7}}\left|f^{\prime \prime}(b)\right|^{q}+\frac{59}{3^{5} 2^{7}}\left|f^{\prime \prime}(a)\right|^{q}\right)^{\frac{1}{q}}\right\} \\
& =(b-a)^{2}\left(\frac{1}{162}\right)^{1-\frac{1}{q}} \\
& \left\{\left(\frac{59}{3^{5} 2^{7}}\left|f^{\prime \prime}(b)\right|^{q}+\frac{133}{3^{5} 2^{7}}\left|f^{\prime \prime}(a)\right|^{q}\right)^{\frac{1}{q}}+\left(\frac{133}{3^{5} 2^{7}}\left|f^{\prime \prime}(b)\right|^{q}+\frac{59}{3^{5} 2^{7}}\left|f^{\prime \prime}(a)\right|^{q}\right)^{\frac{1}{q}}\right\}
\end{aligned}
$$

where we use the fact that

$$
\int_{0}^{\frac{1}{2}}\left|\frac{t}{2}\left(\frac{1}{3}-t\right)\right| d t=\int_{\frac{1}{2}}^{1}\left|(1-t)\left(\frac{t}{2}-\frac{1}{3}\right)\right| d t=\frac{1}{162} .
$$

The proof is complete.

COROllary 2.6. Let $f: I \subset \mathbb{R} \rightarrow \mathbb{R}$ be twice differentiable mapping on $I^{\circ}$ such that $f^{\prime \prime} \in L_{1}[a, b]$, where $a, b \in I$ with $a<b$. If $f(a)=f\left(\frac{a+b}{2}\right)=f(b)$ and $\left|f^{\prime \prime}\right|^{q}$ is convex on $[a, b]$ and $q \geq 1$, then the following inequality holds:

$$
\begin{aligned}
& \left|\frac{1}{b-a} \int_{a}^{b} f(x) d x-f\left(\frac{a+b}{2}\right)\right| \\
& \quad \leq(b-a)^{2}\left(\frac{1}{162}\right)^{1-\frac{1}{q}} \\
& \left\{\left(\frac{59}{3^{5} 2^{7}}\left|f^{\prime \prime}(b)\right|^{q}+\frac{133}{3^{5} 2^{7}}\left|f^{\prime \prime}(a)\right|^{q}\right)^{\frac{1}{q}}+\left(\frac{133}{3^{5} 2^{7}}\left|f^{\prime \prime}(b)\right|^{q}+\frac{59}{3^{5} 2^{7}}\left|f^{\prime \prime}(a)\right|^{q}\right)^{\frac{1}{q}}\right\}
\end{aligned}
$$

where $\frac{1}{p}+\frac{1}{q}=1$.

Remark 2.7. By setting $q=1$ in Theorem 2.5 and Corollary 2.6, we obtain Theorem 2.2 and Corollary 2.3 respectively.

COROLlary 2.8. Let $f: I \subset \mathbb{R} \rightarrow \mathbb{R}$ be twice differentiable mapping on $I^{\circ}$ such that $f^{\prime \prime} \in L_{1}[a, b]$, where $a, b \in I$ with $a<b$. If $f(a)=f\left(\frac{a+b}{2}\right)=f(b)$ and $\left|f^{\prime \prime}\right|^{2}$ is convex on $[a, b]$, then the following inequality holds:

$$
\begin{aligned}
& \left|\frac{1}{b-a} \int_{a}^{b} f(x) d x-f\left(\frac{a+b}{2}\right)\right| \\
& \quad \leq(b-a)^{2}\left(\frac{1}{162}\right)^{\frac{1}{2}} \\
& \left\{\left(\frac{59}{3^{5} 2^{7}}\left|f^{\prime \prime}(b)\right|^{2}+\frac{133}{3^{5} 2^{7}}\left|f^{\prime \prime}(a)\right|^{2}\right)^{\frac{1}{2}}+\left(\frac{133}{3^{5} 2^{7}}\left|f^{\prime \prime}(b)\right|^{2}+\frac{59}{3^{5} 2^{7}}\left|f^{\prime \prime}(a)\right|^{2}\right)^{\frac{1}{2}}\right\} .
\end{aligned}
$$




\section{APPLICATIONS TO SPECIAL MEANS}

We shall consider the following special means:

(a) The arithmetic mean: $A=A(a, b):=\frac{a+b}{2}, a, b \geq 0$,

(b) The harmonic mean:

$$
H=H(a, b):=\frac{2 a b}{a+b}, a, b>0,
$$

(c) The logarithmic mean:

$$
L=L(a, b):=\left\{\begin{array}{cc}
a & \text { if } a=b \\
\frac{b-a}{\ln b-\ln a} & \text { if } a \neq b
\end{array}, \quad a, b>0,\right.
$$

(d) The $p$-logarithmic mean

$$
L_{p}=L_{p}(a, b):=\left\{\begin{array}{cl}
{\left[\frac{b^{p+1}-a^{p+1}}{(p+1)(b-a)}\right]^{\frac{1}{p}}} & \text { if } a \neq b \\
a & \text { if } a=b
\end{array}, \quad p \in \mathbb{R} \backslash\{-1,0\} ; a, b>0 .\right.
$$

It is well known that $L_{p}$ is monotonic nondecreasing over $p \in \mathbb{R}$ with $L_{-1}:=L$ and $L_{0}:=I$. In particular, we have the following inequalities

$$
H \leq L \leq A
$$

Now, using the results of Section 2, some new inequalities is derived for the above means.

Proposition 3.1. Let $a, b \in R, 0<a<b$ and $n \in \mathbb{N}, n>2$. Then, we have

$$
\left|\frac{1}{3} A\left(a^{n}, b^{n}\right)+\frac{2}{3} A^{n}(a, b)-L_{n}^{n}(a, b)\right| \leq n(n-1) \frac{(b-a)^{2}}{162}\left[a^{n-2}+b^{n-2}\right] .
$$

Proof. The assertion follows from Theorem 2.2 applied to convex mapping $f(x)=x^{n}, x \in[a, b]$ and $n \in \mathbb{N}$.

Proposition 3.2. Let $a, b \in R, 0<a<b$. Then, for all $q>1$, we have

$$
\begin{aligned}
& \left|\frac{1}{3} H^{-1}(a, b)+\frac{2}{3} A^{-1}(a, b)-L^{-1}(a, b)\right| \\
\leq & (b-a)^{2}\left(\frac{1}{162}\right)^{1-\frac{1}{q}} \\
& \left\{\left(\frac{59}{3^{5} 2^{7}}\left|\frac{2}{b^{3}}\right|^{q}+\frac{133}{3^{5} 2^{7}}\left|\frac{2}{a^{3}}\right|^{q}\right)^{\frac{1}{q}}+\left(\frac{133}{3^{5} 2^{7}}\left|\frac{2}{b^{3}}\right|^{q}+\frac{59}{3^{5} 2^{7}}\left|\frac{2}{a^{3}}\right|^{q}\right)^{\frac{1}{q}}\right\} .
\end{aligned}
$$

Proof. The assertion follows from Theorem 2.5 applied to the convex mapping $f(x)=1 / x, x \in[a, b]$. 


\section{REFERENCES}

M. Alomari, M. Darus and S.S. Dragomir, New inequalities of Hermite-Hadamard type for functions whose second derivatives absolute values are quasi-convex, RGMIA Res. Rep. Coll., 12 (2009), Supplement, Article 17.

[Online:http://www.staff.vu.edu.au/RGMIA/v12(E).asp]

M. Alomari, M. Darus and S.S. Dragomir, New inequalities of Simpson's type for sconvex functions with applications, RGMIA Res. Rep. Coll., 12 (4) (2009), Article 9. [Online:http://www.staff.vu.edu.au/RGMIA/v12n4.asp]

S.S. Dragomir, R.P. Agarwal and P. Cerone, On Simpson's inequality and applications, J. of Inequal. Appl., 5(2000), 533-579.

S. Hussain, M.I. Bhatti and M. Iqbal, Hadamard-type inequalities for s-convex functions I, Punjab Univ. Jour. of Math., Vol.41, pp:51-60, (2009).

B.Z. Liu, An inequality of Simpson type, Proc. R. Soc. A, 461 (2005), 2155-2158.

J. Pečarić, F. Proschan and Y.L. Tong, Convex functions, partial ordering and statistical applications, Academic Press, New York, 1991.

Mehmet Zeki Sarikaya,

Department of Mathematics, Faculty of Science and Arts,

Düzce University, Düzce,

Turkey

e-mail: sarikayamz@gmail.com

Erhan SET,

Department of Mathematics, Faculty of Science and Arts,

Düzce University, Düzce,

Turkey

e-mail: erhanset@yahoo.com

M. Emin Ozdemir,

Atatürk University, K.K. Education Faculty,

Department of Mathematics, 25240, Campus, Erzurum,

Turkey

e-mail: emos@atauni.edu.tr

Received May 2013 